\title{
Perceptually-driven video coding with the Daala video codec
}

Yushin Cho, Thomas Daede, Nathan Egge, Guillaume Martres, Tristan Matthews, et al.

Yushin Cho, Thomas J. Daede, Nathan E. Egge, Guillaume Martres, Tristan Matthews, Christopher Montgomery, Timothy B. Terriberry, Jean-Marc Valin, "Perceptually-driven video coding with the Daala video codec," Proc. SPIE 9971, Applications of Digital Image Processing XXXIX, 997115 (27 September 2016); doi: 10.1117/12.2238417

EDent: SPIE Optical Engineering + Applications, 2016, San Diego, California, United States 


\title{
Perceptually-Driven Video Coding with the Daala Video Codec
}

\author{
Yushin Cho ${ }^{\mathrm{a}, \mathrm{b}}$, Thomas J. Daede ${ }^{\mathrm{a}, \mathrm{b}}$, Nathan E. Egge ${ }^{\mathrm{a}, \mathrm{b}}$, Guillaume Martres ${ }^{\mathrm{a}, \mathrm{b}}$, Tristan \\ Matthews $^{\mathrm{a}, \mathrm{b}}$, Christopher Montgomery ${ }^{\mathrm{a}, \mathrm{b}}$, Timothy B. Terriberry ${ }^{\mathrm{a}, \mathrm{b}}$, and Jean-Marc Valin ${ }^{\mathrm{a}, \mathrm{b}}$ \\ ${ }^{a}$ Xiph.Org Foundation, 21 College Hill Road, Somerville, MA 02144, USA \\ bMozilla Corporation, 331 E. Evelyn Ave., Mountain View, CA 94041, USA
}

\begin{abstract}
The Daala project is a royalty-free video codec that attempts to compete with the best patent-encumbered codecs. Part of our strategy is to replace core tools of traditional video codecs with alternative approaches, many of them designed to take perceptual aspects into account, rather than optimizing for simple metrics like PSNR. This paper documents some of our experiences with these tools, which ones worked and which did not. We evaluate which tools are easy to integrate into a more traditional codec design, and show results in the context of the codec being developed by the Alliance for Open Media.
\end{abstract}

Keywords: AOM, AV1, Daala, compression, codec, video, perceptual

\section{INTRODUCTION}

Designing a royalty-free video codec is a challenging problem. ${ }^{1}$ Using tools and techniques that differ substantially from those used by the common commercially-licensed codecs can reduce that challenge. Over the last several years in the Daala project, ${ }^{2}$ we have experimented with a number of such tools. Some techniques were long known by other researchers, ${ }^{3-5}$ but not commonly used. However, we thought they might not have been sufficiently developed at the time to be competitive with the usual approaches, or might be worth re-evaluating in a modern context due to changes in engineering trade-offs. Other techniques we developed ourselves, ${ }^{6-8}$ either to solve existing problems in new ways or to solve new problems raised by the other tools we chose.

Some of these techniques have been successful, while others did not pan out as well as we hoped. As effort is now underway at the Alliance for Open Media (AOM) to create a royalty-free video codec based on the technologies of many industry partners, including Daala, it is worth analyzing these techniques in that context. The Alliance is starting development of its first codec, AV1, from VP9, ${ }^{9}$ a more traditional codec design, and also has access to patent rights that we did not as part of the Daala project. However, we think that some of the technology we created and many of the lessons we learned can usefully contribute to this effort.

All of the code from the various experiments presented below is available in either the Daala git repository ${ }^{10}$ or the AOM git repository, ${ }^{11}$ except where, as noted, it was done in an author's personal (but still public) repository. All metrics results for Daala are taken from our Are We Compressed Yet (AWCY) website, using the ntt-short-1 sequences and "Entire Curve (old method)" approach for computing the average change in rate at equivalent quality ${ }^{12}$ (except as noted). It makes use of four objective metrics, whose implementations are available in the tools directory of the Daala git repository, which are PSNR, SSIM, ${ }^{13}$ PSNR-HVS-M, ${ }^{14}$ and multiscale FastSSIM. ${ }^{15}$ Metric results for AV1 are also taken from AWCY, but using the newer objective-1-fast test set and the "Report (Overlap)" approach for computing rate changes, and use regular MS-SSIM. ${ }^{16}$ This is the methodology currently proposed in the testing draft of the Internet Engineering Task Force (IETF)'s NETVC working group. ${ }^{17}$ The source code for Are We Compressed Yet is also available. ${ }^{18,19}$

Copyright 2016 Mozilla Foundation. This work is licensed under CC-BY 4.0.

Send correspondence to tterribe@xiph.org.

Applications of Digital Image Processing XXXIX, edited by Andrew G. Tescher, Proc. of SPIE Vol. $9971,997115 \cdot$ C 2016 SPIE · CCC code: 0277-786X/16/\$18 · doi: 10.1117/12.2238417 


\section{AN ANALYSIS OF DAALA CODING TOOLS}

While Daala uses the traditional hybrid video coding model of motion-compensated prediction followed by blockbased transform coding, it differs in most of the details. It uses lapped transforms ${ }^{20}$ instead of an adaptive loop filter for deblocking, with intra prediction performed in the frequency domain (see Section 2.2) and with variableblock-sized Overlapped Block Motion Compensation (OBMC) to eliminate blocking artifacts from the prediction stage. ${ }^{6}$ It quantizes transform coefficients with a technique we term Perceptual Vector Quantization ${ }^{7}$ and codes them with a non-binary multiply-free arithmetic $\operatorname{coder}^{21}$ based on the work of Stuiver and Moffat. ${ }^{4}$ An analysis on the current performance, challenges, and future potential of each of the major tools follows.

\subsection{Lapped Transforms}

Viewed in isolation, lapped transforms are extremely effective. They provide better energy compaction than the DCT with less computational cost than wavelets with similar energy compaction, ${ }^{5}$ while structurally eliminating blocking artifacts (one of the most annoying visual artifacts at low rates) at the cost of some increased ringing. Virtually every transform-based audio codec since MP3 has been built on top of them, yet the only major video codec standard to use them has been VC-1, and then only in intra frames.

Unfortunately, a transform is just one part of a video codec. Conceptually, lapped transforms are a very simple change to the standard block-based design: the adaptive loop filter is replaced by an invertible, nonadaptive post-filter, and the encoder runs the inverse as a pre-filter before a normal DCT and quantization stage. However, in practice they introduce structural complications elsewhere in the design.

1. They cannot be used with standard spatial intra prediction, because the final pixel values of neighboring blocks are not available until after the post-filter is applied. See Section 2.2 for how we attempt to address this.

2. They make the inter/intra mode decision problem more complicated. See Section 2.3 for a discussion of this issue.

3. As originally proposed, ${ }^{22}$ they introduce $2 \mathrm{D}$ dependencies into the transform block size decision, which makes the problem NP-hard.

4. They are less localized than a DCT, which makes localized changes in inter frames more difficult to code. This is not an issue for still images, because intra prediction is generally less effective, and the content which must be coded by the transform stage is less localized.

In theory, many of these are also true for an adaptive loop filter. The final pixel values for a neighboring block are not available until after it runs, the size of the filter and the order it is applied may depend on the choice of a neighbor's block size (causing the same 2D dependency problems), and when prediction errors are present on only one side of a block edge, they can introduce spurious changes into pixels on the other side. However, a typical encoder ignores all of these effects, trusting that it can simply optimize the output of the transform and quantization stage. This assumes that because the loop filter is adaptive, it will not make the result worse (or not enough to matter). With lapped transforms, the post-filter is not adaptive. It gets applied even when there is little to no horizontal or vertical correlation across a transform block edge. That makes these issues impossible to ignore.

\subsubsection{Filter Ordering and Block Size Decision}

The 2D dependency problem is perhaps the most severe. In our original design, we apply the post-filter in the same order as a traditional adaptive loop filter. We apply the post-filter to each row of pixels that crosses a vertical block edge first (see Figure 1c), followed by each column of pixels that crosses a horizontal block edge (see Figure 1d). This maximizes parallelism (all row filters can be applied in parallel, followed by all column filters) and minimizes the buffering required. The pre-filter is applied in the opposite order. The size of the pre-/postfilter for each edge is the maximum that does not penetrate more than halfway into any neighboring transform block. That is, we use the largest overlap we can apply that will not introduce any additional dependencies on 
the order the filters are applied, under the constraint that the overlap size is constant for a whole transform block edge. E.g., a $16 \times 16$ transform block next to an $8 \times 8$ transform block and two $4 \times 4$ transform blocks uses a 4-tap filter along the entire shared edge (see Figure 1c). We term this scheme the "full lapping" scheme.

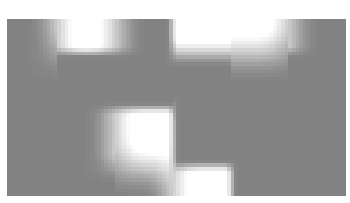

(a) DC basis functions.

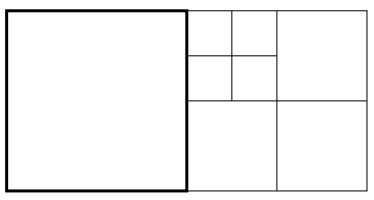

(b) Example block sizes.

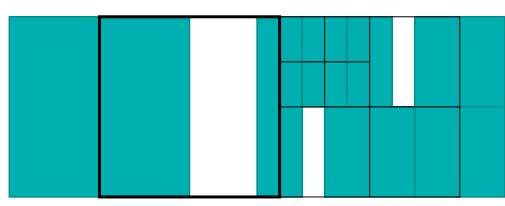

(c) Lapping vertical edges.

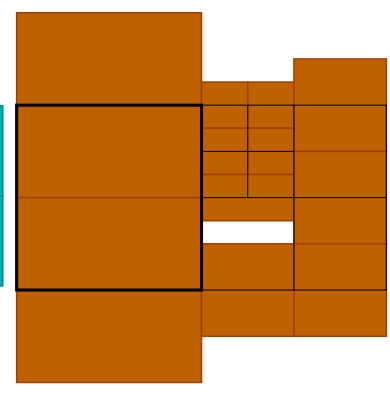

(d) Horizontal edges.

Figure 1: Example DC basis functions for randomly chosen transform block sizes (a) and an (unrelated) example block size partitioning along with the vertical and horizontal lapping applied to each edge with the full lapping scheme (b-d).

This scheme has two issues. The first is that the filtering order can produce some very strangely shaped basis functions. To demonstrate this, we generated a frame with randomly chosen block sizes and set the DC coefficient in some blocks to a large, positive value. The result can be seen in Figure 1a. Although disconcerting, we never observed visual artifacts in real images with similar patterns, so this problem is mostly theoretical (and one that affects adaptive loop filters, as well).

The second problem is the much more practical issue that we have no idea how to search for the optimal transform block size. In order to compute the rate and distortion required for a traditional rate-distortion (RD) search, we needed to know what filters were applied along each edge, which requires knowing the size of neighboring transform blocks. It is not even enough to know the size of the lapping on each edge. If a neighbor on the other side of a vertical edge is split, then the pre-filter is applied along the horizontal edge between them before it is applied along the vertical edge in the current block, changing the filter output. That is, in the example of Figure 1b, the pre-filters in Figure 1d are applied before the pre-filters in Figure 1c. Therefore, it is necessary to know which neighboring block is $8 \times 8$ and which are $4 \times 4$ to properly determine the output of the pre-filter in the $16 \times 16$ block. The corresponding dependency graph does not have a tree structure which admits a simple dynamic programming solution.

Our initial solution to this was to use a heuristic to select block sizes up-front, based on a perceptual estimate of the visibility of ringing artifacts. While this seemed to do vaguely reasonable things for intra frames, we did not have a known-optimal algorithm to compare it to, so we could not be certain how far off it really was. We were able to determine it was doing a poor job for inter frames: simply biasing the decision away from $4 \times 4$ and $8 \times 8$ blocks for large quantizers reduces the bitrate by more than $5 \%$ on all of the metrics we tested*.

\subsubsection{Fixed Lapping}

In order to permit a true RDO search, we change two things. ${ }^{23}$

First, we change the lapping order. Dai et al. propose filtering on $16 \times 16$ macroblock $(\mathrm{MB})$ boundaries first, followed by interior block edges (using the same order as the full lapping scheme in the interior). ${ }^{22}$ During block size decision, they use the smallest possible pre-filter on MB boundaries, and then exhaustively search partitions of the interior using the maximum possible overlap on internal block boundaries (as we described above). After block size decision, in a second pass, they filter MB boundaries with the maximum possible overlap for actual encoding, as in our full lapping scheme. In their implementation, MBs can be split down to $4 \times 4$, producing a total of 17 possible quad-tree subdivisions, which is still tractable to search exhaustively. However, at the time

\footnotetext{
${ }^{*}$ See commit be43a7c18ea7 in the Daala git repository ${ }^{10}$
} 
we conducted these experiments, we already supported block sizes up to $32 \times 32$, which would require searching 83,522 partitions, and would later extend this to $64 \times 64$ (more than 48 quintillion partitions).

Instead, we apply what they do on MB boundaries recursively. Starting at the largest block size, what we term a superblock (SB), we pre-filter the exterior SB edges first. If the block is split, then we pre-filter the interior edges, and then recurse into each quadrant. Again, when post-filtering, we reverse the order.

This reduces the amount of potential parallelism, but still allows filtering all SB interiors in parallel, followed by all vertical SB edges in parallel and then all horizontal SB edges. But this change alone already makes our problem simpler. Returning to the example of Figure 1b, we no longer need to know which adjacent blocks are $4 \times 4$, since the edge between them will always be pre-filtered after the edge they share with the $16 \times 16$ block. All that remains is the issue of the filter size.

To solve this problem, we make our second change: we make the filter size constant. At first we used 8-point filters until we had split blocks down to $4 \times 4$, and then used 4-point filters on the final interior edges. However, currently we use 4-point lapping everywhere, as it provides substantial reductions in ringing, at the cost of some blocking artifacts and some detail preservation. We term this the "fixed lapping scheme", illustrated in Figure 2.

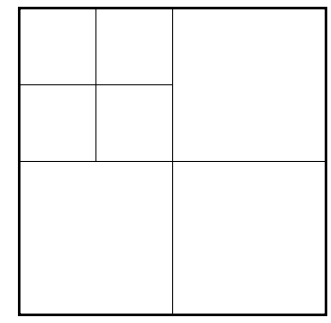

(a) Example block sizes.

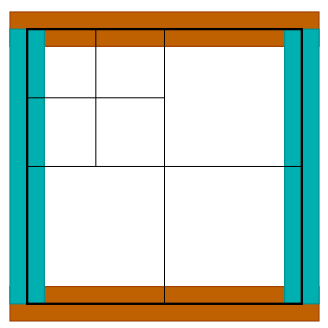

(b) Exterior edges.

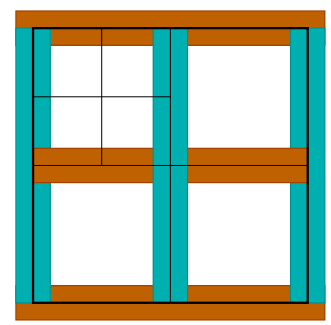

(c) Interior edges.

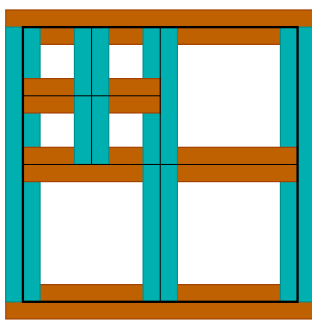

(d) Recursion.

Figure 2: Example demonstrating the pre-filtering order for the fixed lapping scheme. At each step, the horizontal edges are pre-filtered before the vertical ones, to minimize buffering when applying the post-filter.

With those two changes, it is now possible to compute R-D optimal transform block sizes. After pre-filtering the exterior edges, we try splitting the block and pre-filtering the interior edges. Then we compute the optimal partitioning of each of the four quadrants (recursively), then apply the post-filter to the interior edges. At this point, although we have not post-filtered the exterior edges, it is still possible to do an apples-to-apples comparison of the distortion between the split and no-split cases.

The result is large rate reductions on all metrics: $10.3 \%$ and $12.3 \%$ on PSNR and SSIM, respectively, and $4.5 \%$ and $5.2 \%$ on PSNR-HVS and Fast MS-SSIM, respectively, compared to the full lapping scheme ${ }^{\dagger}$. For intra frames, the results showed similarly large gains, indicating that even though our heuristic looked "vaguely reasonable" in this case, it was not actually doing a good job. We also experimented with an approach similar to Dai et al.'s, where block size decisions are made using the fixed lapping size, but the actual video is encoded with the full lapping sizes (though still applied in recursive order). When optimizing for PSNR (using flat quantization and no activity masking), this also achieves large gains: a $9.2 \%$ reduction in bitrate ${ }^{\ddagger}$. This was an encoder-only change, and we verified the bitstreams were decodable by the mainline decoder at the time. This shows that most of the improvements of the fixed-lapping approach simply came from making better decisions than our previous heuristics. However, we opted to keep the fixed lapping size. The gains with the full lapping size are not as large as when using fixed lapping, and it is not obvious how to tune such an encoder, since the actual data that gets encoded can be significantly different than what is used to make decisions.

${ }^{\dagger}$ With the initial 8-point lapping. See commits 34b6d5950541 through 1a606b2c8e63 in the Daala git repository. ${ }^{10}$ This also included the addition of a bilinear smoothing filter on intra frames, ${ }^{23}$ to reduce the blocking artifacts caused by the reduction in lapping. Despite visual reductions in blocking, it actually slightly reduced metrics, and was later removed after it was found that $64 \times 64$ DCTs and our deringing filter provided the same benefits, and it was no longer helping on most images. See commit eca1b5a207bf.

${ }^{\ddagger}$ See the var_lap_jm_exp_psnr2 branch of Yushin Cho’s personal Daala repository: https://git.xiph.org/users/ yushin/daala.git. 
It might also be possible to explicitly signal the size of the pre-filters to use. The search remains tractable in the interior of a superblock, but some kind of heuristic would be required for the lapping on edges between superblocks, as they retain the original 2D dependency problem. Given that most of the motivation for larger lapping is the reduction in blocking artifacts in large regions that are either smooth or uniformly textured (where the largest transform blocks are used), this is a problem that would need to be solved. This is an area we have not explored.

\subsubsection{Lapping vs. Loop Filters}

The same recursive filter application order can also be applied to a standard adaptive loop filter. This allows an encoder to consider the effect of the loop filter when making transform block size decisions. We modified Daala to use the adaptive loop filter from the Thor video $\operatorname{codec}^{24}$ in place of lapping through a compile-time option. When enabled, this produces bitrate reductions between $0.4 \%$ and $2.6 \%$ on video, depending on the metric used ${ }^{\S}$. However, when used on still images it produces bitrate increases between $2.5 \%$ and $3.2 \%$ (with the exception PSNR-HVS, where bitrate falls by $0.3 \%$. The visual impact is more difficult to judge. We can also get metrics gains on video by disabling lapping completely, despite the obvious blocking and loss of detail preservation that causes. However, we have not attempted to do a formal subjective comparison between lapped transforms and adaptive loop filters, and the results would probably be too close to justify the time and effort. So, although we believe we have made good strides in understanding how to use lapping in a modern video codec, it is not obviously technically superior.

In the context of the Alliance for Open Media, using lapping would require so many structural changes to the codec that it would not be possible to do and still meet our time-to-market goals, so it is not currently being pursued. However, the recursive filter ordering may still provide some benefit in terms of improved encoder decisions and potentially eliminating artifacts along the lines of Figure 1a. This is something we hope to experiment with in the future.

\subsection{Frequency-Domain Intra Prediction}

There are ample reasons in the research literature to suggest that the issue of intra prediction with lapped transforms raised in Section 2.1 is a solvable problem. For example, de Oliveira and Pesquet-Popescu show gains on five images by integrating lapped transforms into a version of H.264/AVC restricted to $8 \times 8$ blocks and performing intra prediction from four pixels farther away. ${ }^{25}$ This is a promising result, but raises several practical issues. First, they restrict the prediction to four of nine intra modes in the lapped transform case, because they do not have sufficient pixels available for the other modes. Second, determining which pixels are available for prediction becomes much more complicated when introducing multiple block sizes, especially with the full lapping scheme (which we were still using when performing most of our intra experiments). This approach also has a significant impact on the potential parallelism, since enough post-filtering must be applied to make the required pixels available before the current block can be reconstructed (and then post-filtered), but this is a problem with spatial intra prediction generally.

We draw inspiration from $\mathrm{Xu}$ et al., who recharacterize traditional spatial-domain intra prediction as an "intra-predictive transform": ${ }^{26}$

$$
\left[\begin{array}{l}
\tilde{\mathbf{x}} \\
\mathbf{y}
\end{array}\right]=\left[\begin{array}{cc}
\mathbf{I} & \mathbf{0} \\
\mathbf{0} & \mathbf{D C T}
\end{array}\right]\left[\begin{array}{cc}
\mathbf{I} & \mathbf{0} \\
-\mathbf{E} & \mathbf{I}
\end{array}\right]\left[\begin{array}{l}
\tilde{\mathbf{x}} \\
\mathbf{x}
\end{array}\right]
$$

Here, $\tilde{\mathbf{x}}$ represents the pixels used as predictors, which are unchanged by the transform, and $\mathbf{E}$ represents the prediction process. In the simplest case, where $\tilde{\mathbf{x}}$ is a single pixel, $\mathbf{E}$ might be a column of 1 s. Then $\mathbf{y}$ would be the DCT of the residual after extending $\tilde{\mathbf{x}}$ into the block and subtracting it from each pixel in $\mathbf{x}$.

\footnotetext{
${ }^{\S}$ See commit ae4a21325d62 in the Daala git repository. ${ }^{10}$

"Using the same commit. Tested on subset1, a set of 50 images sampled from Wikipedia and downsampled to about one megapixel on AWCY.
} 
This approach can be extended to move the prediction into the frequency domain. We can apply any invertible transform to $\tilde{\mathbf{x}}$ (for example, the DCT) and still achieve identical output for $\mathbf{y}$ by applying the inverse transform to $\mathbf{E}$. That is,

$$
\left[\begin{array}{l}
\tilde{\mathbf{y}} \\
\mathbf{y}
\end{array}\right]=\left[\begin{array}{cc}
\mathbf{I} & \mathbf{0} \\
\mathbf{0} & \mathbf{D C T}
\end{array}\right]\left[\begin{array}{cc}
\mathbf{I} & \mathbf{0} \\
-\mathbf{E} \cdot \mathbf{D C T} \mathbf{T}^{-1} & \mathbf{I}
\end{array}\right]\left[\begin{array}{cc}
\mathbf{D C T} & \mathbf{0} \\
\mathbf{0} & \mathbf{I}
\end{array}\right]\left[\begin{array}{l}
\tilde{\mathbf{x}} \\
\mathbf{x}
\end{array}\right]
$$

Multiplying together the first two matrices on the right hand side produces

$$
\left[\begin{array}{l}
\tilde{\mathbf{y}} \\
\mathbf{y}
\end{array}\right]=\left[\begin{array}{cc}
\mathbf{I} & \mathbf{0} \\
-\mathbf{D C T} \cdot \mathbf{E} \cdot \mathbf{D C T} \mathbf{T}^{-1} & \mathbf{D C T}
\end{array}\right]\left[\begin{array}{cc}
\mathbf{D C T} & \mathbf{0} \\
\mathbf{0} & \mathbf{I}
\end{array}\right]\left[\begin{array}{l}
\tilde{\mathbf{x}} \\
\mathbf{x}
\end{array}\right] .
$$

Then, observing that we can also pre-transform $\mathbf{x}$ if we correct for it in the next step we have

$$
\left[\begin{array}{l}
\tilde{\mathbf{y}} \\
\mathbf{y}
\end{array}\right]=\left[\begin{array}{cc}
\mathbf{I} & \mathbf{0} \\
-\mathbf{D C T} \cdot \mathbf{E} \cdot \mathbf{D C T}^{-1} & \mathbf{D C T}
\end{array}\right]\left[\begin{array}{cc}
\mathbf{I} & \mathbf{0} \\
\mathbf{0} & \mathbf{D C T}^{-1}
\end{array}\right]\left[\begin{array}{cc}
\mathbf{I} & \mathbf{0} \\
\mathbf{0} & \mathbf{D C T}
\end{array}\right]\left[\begin{array}{cc}
\mathbf{D C T} & \mathbf{0} \\
\mathbf{0} & \mathbf{I}
\end{array}\right]\left[\begin{array}{c}
\tilde{\mathbf{x}} \\
\mathbf{x}
\end{array}\right],
$$

which simplifies to

$$
\left[\begin{array}{l}
\tilde{\mathbf{y}} \\
\mathbf{y}
\end{array}\right]=\left[\begin{array}{cc}
\mathbf{I} & \mathbf{0} \\
-\mathbf{D C T} \cdot \mathbf{E} \cdot \mathbf{D C T} & \mathbf{I}
\end{array}\right]\left[\begin{array}{cc}
\mathbf{D C T} & \mathbf{0} \\
\mathbf{0} & \mathbf{D C T}
\end{array}\right]\left[\begin{array}{l}
\tilde{\mathbf{x}} \\
\mathbf{x}
\end{array}\right]
$$

Letting the matrix $\mathbf{F}=\mathbf{D C T} \cdot \mathbf{E} \cdot \mathbf{D C} \mathbf{T}^{-1}$, we call $\mathbf{F}$ the frequency domain predictor that is equivalent to $\mathbf{E}$. For any spatial domain predictor $\mathbf{E}$, we can derive an equivalent frequency-domain predictor $\mathbf{F}$ by passing it through the corresponding transforms.

Since we know that lapped transforms have similar frequency-domain behavior to that of the DCT, generally, we posit that if we replace the DCT by a lapped transform, there exist similar frequency-domain predictors. We can no longer claim direct equivalence to a spatial-domain predictor, and it is not at all clear that the resulting predictors admit efficient implementations. Instead of attempting to design such predictors theoretically, we train them from real data, following an approach similar to that used for directional transforms by Sezer et al. ${ }^{27}$

We classify blocks on a set of 1,000 natural images sampled from Wikipedia (subset3 on AWCY), each downsampled to about one megapixel, using the ten intra prediction modes from VP8, ${ }^{28}$ extended to work with arbitrary block sizes, picking the one that minimizes the Sum of Absolute Transformed Difference (SATD). Then, we train ten frequency domain predictors using least-squares regression on the blocks for each mode, and re-classify the blocks using the new predictors, and iterate this process, as illustrated in Figure 3. Initially we weighted the blocks based on how well the best predictor fared compared to the second best, in order to reduce the impact of outliers, blocks which were either equally well predicted by several modes, or blocks not well predicted by any mode. However, this did not appear to help, and we eventually disabled it.

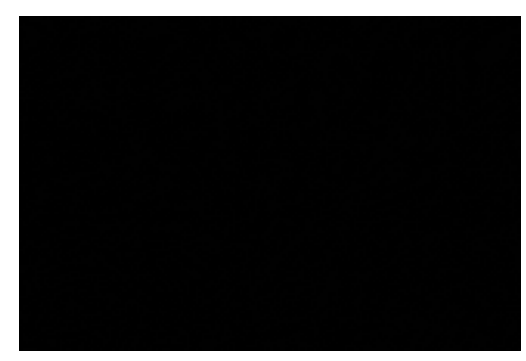

(a) Original image.

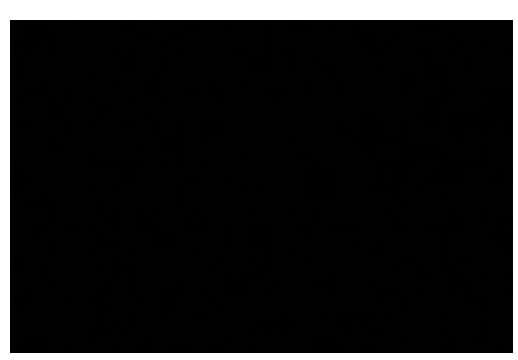

(b) VP8 intra classification.

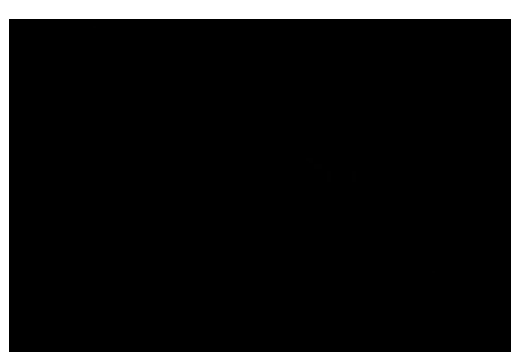

(c) After K-means iteration.

Figure 3: Classification of blocks by intra mode during training. (a) shows the original image, followed by the classification of $4 \times 4$ blocks by their best intra prediction mode (b) using the VP8 predictors and (c) after several iterations of training. Black represents the DC mode, white the True Motion mode, and the remaining colors the directional modes, arranged by hue. 
Beyond the initial classification using the VP8 intra predictors, we imposed no constraint to ensure that the predictors remained directional, though they generally did soll. Interestingly, one mode did diverge significantly from its original use: VP8's "True Motion" mode. This mode forms its prediction as $p_{i j}=U_{j}+L_{i}-U L$ where $(i, j)$ is the coordinate of the pixel being predicted, $U_{j}$ is the corresponding pixel on the edge of the neighboring block above, $L_{i}$ is the corresponding pixel on the edge of the neighboring block to the left, and $U L$ is the pixel at the corner of the neighbor above and to the left. While in VP8 its primary utility is in predicting gradients, after training we found that it was used in highly textured regions, as illustrated in Figure 4. Closer inspection suggests that what the mode is actually doing is copying the contents of its neighboring blocks at a half-cycle offset (that is, at an offset half the size of the block). It seems possible to manually design such an intra mode, in either the frequency or the spatial domain, though we are not aware of anyone doing so.

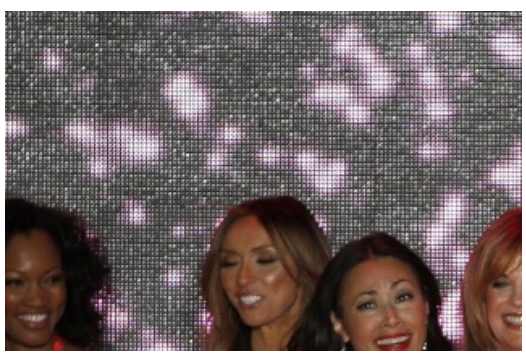

(a) Original image.

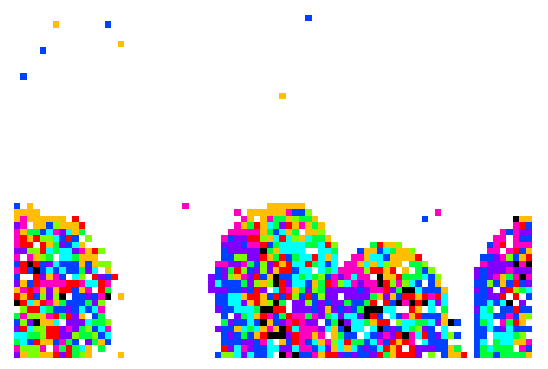

(b) Intra classification.

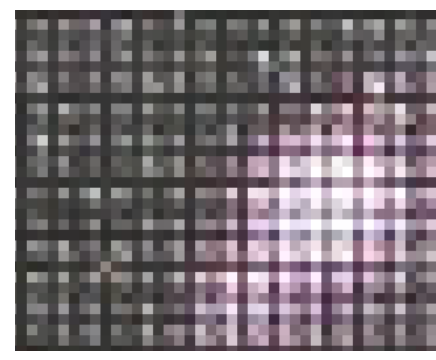

(c) Close-up of texture.

Figure 4: An image with a patterned background texture. (a) A cropped region of the original image, (b) the corresponding classification by intra modes after training, and (c) a close-up of the background texture pattern. Intra mode colors are the same as in Figure 3.

In order to ensure that our predictors are computationally feasible, we set a complexity budget of four multiplies per output coefficient. We force a fixed number of additional entries in the prediction matrix $\mathbf{F}$ to zero at each iteration until this budget is met. We experimented with picking the smallest entries to force to zero or picking the ones that had the smallest impact on prediction gain (that is, that gave the smallest increase in the geometric mean of the variance of the prediction residuals), and the latter, though much slower to train, appears universally better (see Figure 5).

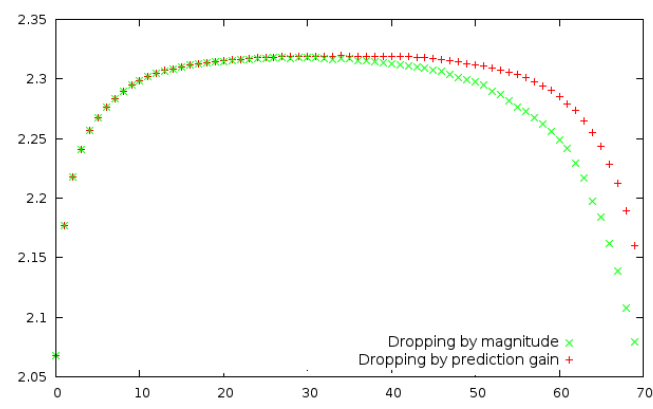

(a) Average prediction gain $(\mathrm{dB})$.

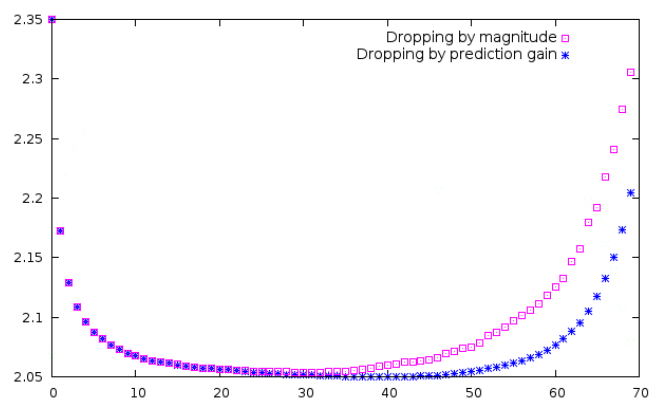

(b) Estimated lossless bitrate (bits per pixel).

Figure 5: Comparison of coefficient zeroing strategies at each iteration $(x$ axes) of predictor training for $4 \times 4$ blocks on 50 images. Estimated bitrates are based on the entropy of coding Laplacian distributions with a known variance and do not include the cost to code the prediction modes.

We ran this procedure, with and without the sparsity constraint, on our 1,000 image training set using fixed $4 \times 4,8 \times 8$, and $16 \times 16$ transform block sizes, and compared the prediction gain to the spatial domain VP8 predictors with the DCT. The results are summarized in Table 1. Coding gain and prediction gain are computed

\footnotetext{
"See https://people.xiph.org/ xiphmont/demo/daala/demo2.shtml for an interactive demonstration of the $4 \times 4$ predictors.
} 


\begin{tabular}{lrrrrrrrrrr}
\hline & \multicolumn{3}{c}{ DCT+VP8 Intra } & & \multicolumn{2}{c}{ LT+FDIP (full) } & & \multicolumn{2}{c}{ LT+FDIP (sparse) } \\
\cline { 2 - 3 } Transform size & $C_{g}$ & $P_{g}$ & Total & & $C_{g}$ & $P_{g}$ & Total & & $P_{g}$ & Total \\
\hline $4 \times 4$ & 13.8511 & 2.9154 & 16.7665 & & 14.9600 & 2.2103 & 17.1703 & & 2.0799 & 17.0399 \\
$8 \times 8$ & 15.1202 & 0.86940 & 15.9894 & & 15.6468 & 0.72486 & 16.3716 & & 0.58957 & 16.2364 \\
$16 \times 16$ & 15.5870 & 0.19483 & 15.7818 & & 15.8721 & 0.37593 & 16.2480 & & 0.09541 & 15.9675 \\
\hline
\end{tabular}

Table 1: Coding gains and prediction gains of the DCT with the VP8 intra predictors compared to lapped transforms and frequency-domain intra prediction (FDIP) with (full) and without (sparse) the predictor sparsity constraint. The "Total" columns represent the coding gain of the corresponding transform applied to the prediction residual. All numbers are in decibels (dB, higher is better).

using actual measured variances of the full 2D transform coefficients, rather than the traditional first-order auto-regressive $\operatorname{AR}(1)$ model with correlation coefficient $\rho=0.95$.

At the $4 \times 4$ level, the sparse predictors work fairly well, with little loss relative to the non-sparse versions. The frequency-domain predictors show less prediction gain than their spatial counterparts, but this is because some of the correlation with neighboring blocks has already been removed by the lapping, which is accounted for in the higher coding gains of the lapped transforms. The prediction gain for the full $16 \times 16$ predictor actually appears to be higher than that of the spatial predictor, but at this size each prediction matrix has 262, 144 free parameters. With only 1,000 one megapixel training images, and ten modes to train, this gives less than two $16 \times 16$ input image blocks per free parameter, so this result is probably due to over-fitting. Sparsification reduces this to only 1,024 free parameters per prediction mode, but this also eliminates most of the prediction gain.

Overall, the prediction gain for sizes larger than $4 \times 4$ is disappointing, and the sparsity constraint has a fairly significant impact on the prediction quality. In practice, we found that the training would spend a large portion of its non-zero matrix entries predicting DC, as this had the biggest impact on the prediction gain. This was unsatisfying, as there are less expensive ways to predict DC coefficients (see Section 2.2.2).

\subsubsection{Variable Block Sizes}

Extending this to variable block sizes creates even more complications. All of the prediction matrices trained above assume that each block is the same size as its neighbors. In practice, they may be different sizes. Training separate prediction matrices for every potential combination of neighboring block sizes is clearly impractical.

To handle this, we borrow a tool from the Opus audio codec: Time-Frequency (TF) resolution switching. ${ }^{29}$ The basic idea is to apply a Walsh-Hadamard Transform (WHT) to very cheaply merge multiple blocks of frequency-domain coefficients into a single, larger block with higher frequency resolution, or conversely to split a large block of frequency-domain coefficients into smaller blocks with higher time resolution. The conversion is grossly approximate, but can be "good enough" for some purposes.

Since all of our coefficient blocks are two-dimensional (unlike audio), we can implement a particularly cheap integer version that is a has orthonormal scaling (i.e., is $L_{2}$-norm preserving) and exactly reversible, with just seven additions and one shift. In order to implement the $2 \times 2 \mathrm{WHT}$

$$
\left[\begin{array}{ll}
A & B \\
C & D
\end{array}\right]=\frac{1}{2}\left[\begin{array}{cc}
1 & 1 \\
1 & -1
\end{array}\right]\left[\begin{array}{ll}
a & b \\
c & d
\end{array}\right]\left[\begin{array}{cc}
1 & 1 \\
1 & -1
\end{array}\right]
$$

we compute

1. $e=a+c, f=d-b$,

2. $g=\frac{1}{2}(e-f)$,

3. $B=g-b, C=g-c$,

4. $A=e-B, D=f+C$. 
This is significantly cheaper than other approaches described in the literature, such as that of Fukuma et al. ( 8 additions, 2 shifts, and 1 negation $)^{30}$ or that used by JPEG XR (10 additions, 1 shift, and 2 negations), ${ }^{31}$ and is, to our knowledge, a novel result.

With this tool in hand, we can handle variable block sizes several ways. For example, for each transform block, we can apply TF merging or splitting until our neighbors are all the same size as the current block, and then use the predictors trained for that size. This is what we implemented first.

However, there is no requirement that our predictors be trained with neighbors that are the same size as the block being predicted. Instead, we can TF all of our neighbors down to $4 \times 4$, and then train predictors for each block size from the TF'd $4 \times 4$ data. During training, we transform all blocks at a fixed block size, and then $\mathrm{TF}$ the transform coefficients in a block's neighbors from that size down to $4 \times 4$. We also tried using the block size heuristic from Section 2.1.1 to choose block sizes, limiting the training for each size to blocks that would be encoded at that size, but this seemed to have little impact. Using TF'd $4 \times 4$ neighbors has a number of advantages:

1. Much simpler logic, since everything is converted to the same size, and we don't have to retransform the same blocks into multiple different TF resolutions when using them to predict multiple neighbors of different sizes.

2. Reduced memory footprint, since we only have to buffer one row of $4 \times 4$ coefficient values, rather than all coefficients for an entire row of superblocks.

3. Less overfitting during training, since at $16 \times 16$ we only have 53,248 free parameters per prediction mode (before sparsification) instead of 262, 144 .

The difference between the two approaches on metrics is small. The TF' $4 \times 4$ approach gives a tiny gain on PSNR and loses between $0.4 \%$ and $1.4 \%$ on our other metrics**, but considering its other advantages we thought those losses acceptable.

\subsubsection{A Simpler Approach}

Although we are able to build a practical codec with lapped transforms and variable block sizes based on the frequency-domain intra prediction scheme described in the previous sections, we cannot say that it truly works well. The promising results of images coded at a fixed $4 \times 4$ block size disappear when larger block sizes are allowed, and over-fitting and training time, already a problem with $16 \times 16$ transform blocks, pose a serious challenge for $32 \times 32$ and above. The prediction does show gains of a few percent on metrics, but not enough to justify the computational complexity of the approach: a sparse matrix multiply with four multiplies per predicted coefficient, multiplied by the number of modes that have to be searched in the encoder, with a large table of trained prediction parameters, and a row buffer of $4 \times 4 \mathrm{TF}^{\prime} \mathrm{d}$ coefficient values (four times the size of the equivalent buffer for a spatial predictor). It also has the disturbing tendency to occasionally produce large, erroneous, isolated high-frequency coefficients (likely due to over-fitting in the training), which nevertheless cannot be corrected at low rates, an artifact we term "basis break-out".

It is not obvious how to improve performance. Better training and more training data could improve the quality of the predictors we obtain, but we tried many different variations of our training methods, and suspect the potential upside here is limited. The approximations in TF are certainly one potential culprit. Although TF itself is orthonormal, our lapped transforms are biorthogonal, and each basis function has a different magnitude, depending on which post-filters are applied. With the full lapping scheme, it is difficult to even compute the magnitudes of the basis functions, as the filter order makes them depend on the size of all of ones neighbors (see Figure 1a). That makes it impractical to correct for the magnitudes of basis functions obtained via TF compared to those obtained with a fixed block size, much less for inaccuracies in the shape of the basis functions. That limits the effectiveness of the predictors we can train. With the fixed lapping scheme, these issues may be worth revisiting, as might the approach of de Oliveira and Pesquet-Popescu.

\footnotetext{
${ }^{* *}$ See commit 2b03b324f7d6 in the Daala git repository. ${ }^{10}$ These tests were done using the rd_collect.sh script in the tools directory of that repository, since they predated AWCY
} 
Instead, we opt to use two much simpler tools. The first, called Haar DC, ${ }^{32}$ simply applies our $2 \times 2$ WHT to recursively merge just the DC coefficients from each block up to the superblock level. At the superblock level, DC is predicted using a simple, static linear predictor. This ensures that we retain fine quantization of DC over large regions, since the magnitude of the DC basis function doubles with each application of the WHT, but the quantizer resolution does not. It also greatly reduces the number of bits spent on DC.

The second simply copies the first row and/or column of AC coefficients from its neighbors, much like MPEG4 Part 2, with the important distinction that we use the copied coefficients as predictors before quantizing the AC coefficients in the current block, not afterwards. This copying is only done when the neighbors use the same transform block size. We experimented with using TF to generate predictors when the transform sizes differed, but this did not produce any appreciable gains.

Since we already partition each block into multiple frequency bands, and signal whether or not to use the prediction in each one (using "noref" flags, see Section 2.4), this approach requires no additional signaling, and no additional searching. For the lowest band, which contains portions of both the first row and the first column of AC coefficients, we only copy the one that has higher energy. On typical images, this prediction only reduces rate by 1 to $2 \%$, but on a synthetically generated image of a large checkerboard, it reduces rate by around $50 \%$. That is, its main utility is to prevent embarrassingly bad performance on trivial images.

Together, these two tools are about as effective as the much more complex frequency-domain intra prediction schemes of the previous sections. In the context of the Alliance for Open Media, frequency-domain intra prediction is mostly unnecessary, since without lapped transforms, spatial intra prediction can be used instead. That means neither Haar DC nor the simple AC coefficient copying are being pursued for integration. However, the pattern predictor illustrated in Figure 4 may have some potential as an additional prediction mode. The difficulty will be justifying the additional line buffers it would require in a hardware implementation.

\subsection{Overlapped Block Motion Compensation}

There are multiple ways to incorporate motion compensation into a codec with lapped transforms. Tran and Tu originally proposed applying the pre-filter to the reference frame, at offsets determined by a motion vector (MV), to produce lapped reference pixels. ${ }^{33}$ However, this requires redundant filtering of edges when neighboring MVs do not match, especially during the encoder's motion search. It also directly ties the prediction block size to the transform block size, a tie that the most recent video codecs, such as Thor ${ }^{24}$ and VP10, ${ }^{34}$ have reduced or eliminated.

Instead, we produce a complete "motion-compensated reference frame", and then apply exactly the same lapping to this frame as we apply to the input image to produce our predictors. The prediction stage is completely decoupled from the transform stage. With this structure, we can use any method we wish to create the motioncompensated reference frame. We choose to use a variable-block-sized Overlapped Block Motion Compensation (OBMC) scheme. ${ }^{6}$ In addition to eliminating blocking artifacts, it also produces a relatively uniform prediction error, which, together with our vastly simplified intra prediction, mostly eliminates the need for tools such as adaptive transform type selection. ${ }^{35}$ We view this as an advantage, as having fewer alternatives allows for less searching at the encoder and a simpler implementation.

The major drawback of this approach is that the motion estimate stage has no way to know when the transform stage will choose to discard the prediction (using "noref" flags, see Section 2.4). This is the closest we have to an intra mode in inter frames, as we restrict the AC copying scheme described in Section 2.2.2 to intra frames for simplicity. In regions that are difficult to predict, it is possible we will waste bits producing a prediction that is not good enough for the transform stage to use.

We have considered a number of ideas for mitigating this problem. We could include using a heuristic to approximate the transform stage's decision, an approach commonly used for mode decision in other codecs. Or, we could use a special reference frame that produces a constant color as its prediction, which would then be easy to blend with the predictions of other reference frames in the OBMC scheme. However, we have not implemented these ideas, and do not know how effective they would be. 
However, one advantage this structure brings is the ability to easily swap out one prediction scheme for another. To compare the objective performance of our OBMC scheme with a traditional Block Matching Algorithm (BMA) implementation, we do just that, using the open-source Thor codec. We simply run the entire Thor encoder with residual coding disabled in place of OBMC, despite the obvious issues with blocking artifacts (although Thor has a loop filter, it only runs when residuals are actually coded). To make the comparison more fair, we disable Thor's intra prediction modes and $64 \times 64$ prediction block sizes (which the version of Daala we tested did not yet support), and stop coding the relevant bits that would have signaled them ${ }^{\dagger \dagger}$. The result was an increase in bitrate of $7.2 \%$ to $9.2 \%$, depending on the metric. However, given the complexity of our OBMC scheme, this was not as large as we were expecting. For comparison, at the time we conducted these experiments, Thor spent approximately $10 \%$ of its encoding time on motion estimation, while Daala spent approximately $70 \%$.

We then add back in Thor's intra modes ${ }^{\ddagger}$. This reduces the rate of using Thor’s MC by $1.6 \%$ to $2.0 \%$ (depending on the metric, FastSSIM actually increased by $0.5 \%$ ), but it is still worse than Daala's. Although this does not completely eliminate the potential to waste bits on predictions that are not used, it gives some idea of the potential effectiveness of mitigation strategies in the motion estimation stage.

While these results are interesting, it is not straightforward to perform the reverse experiment-putting Daala's OBMC scheme into an encoder that tightly couples its search for both optimal predictions and the optimal transform coding of the residual from those predictions. Nor is it clear how to incorporate the usual spatial intra prediction modes into our OBMC scheme without violating our structural guarantees against blocking artifacts (which would defeat the purpose of the scheme). Thus, given how much of the encoder we would have to rewrite and how many coding tools we would have to discard, we are not currently planning to integrate our OBMC scheme into AV1, despite its promise.

\subsection{Perceptual Vector Quantization}

Perceptual Vector Quantization ${ }^{7}$ is a gain-shape quantization technique that also has its origins in the Opus audio codec. $^{29}$ Its primary advantage over scalar quantization is that it offers a lot more flexibility in automatically controlling the allocation of bits. This is due to the fact that it extracts several meaningful parameters from the vector of transform coefficients: both a gain, representing the amount of contrast in the signal, and $\theta$, which represents the angle between the input vector and a prediction, and thus encodes how well the signal is predicted. PVQ then uses algebraic vector quantization to encode the remaining information in the coefficient vector without any redundant degrees of freedom.

Having a meaningful parameter like the gain allows us to implement things like activity masking without sending any side information. The gain directly tells us how much contrast was in the original signal, which gives an indication of how visible quantization artifacts will be. We even split the coefficients into multiple frequency bands, with a separate gain and $\theta$ encoded for each one, which allows finer control of this effect. Having separate bands also allows us to independently signal for each one whether or not to use the predictor, what PVQ terms a "noref" flag for "no reference". Together with $\theta$, this gives a great deal of flexibility in how the predictor is used, particularly when the reliability of the predictor varies by frequency.

Unlike some of the other tools developed for Daala, conceptually PVQ is a fairly straightforward dropin replacement for scalar quantization. In our original published results on PVQ, ${ }^{7}$ we compare it to a fairly unsophisticated implementation of scalar quantization (i.e., one that we wrote ourselves), with encouraging results. In AV1, we are starting from the scalar quantization implementation in VP9, which has matured and been tuned over the course of many years.

We have begun integrating PVQ into AV1 as an optional experiment*. While this integration process touches many parts of the encoder, and is still an ongoing process, with many unknown bugs to find and unexpected interactions to tune, we have already achieved encouraging results. Currently, this integration targets PSNR,

\footnotetext{
${ }^{\dagger \dagger}$ See commit 41f301b1b142 in the exp-thor-mc1 branch of Timothy B. Terriberry's personal Daala repository: https: //git.xiph.org/users/tterribe/daala.git.

${ }^{\ddagger}$ See commit $35766 \mathrm{e} 08483 \mathrm{~d}$ in the same branch.

*Currently in the av1_pvq branch of Yushin Cho's personal AOM git repository: https://github.com/ycho/aom/ tree/av1_pvq
} 
using flat quantization matrices and disabling activity masking. Only once we are confident this works in all configurations will we attempt to enable more advanced features. We also use a monochrome version of the objective-1-fast test sequences, objective-1-smc, since we have not yet properly tuned chroma with PVQ in AV1.

With this configuration, on still images, we show gains from $1.3 \%$ to $2.0 \%$, with the largest gains in PSNR ${ }^{\dagger}$. For video, we currently see $0.65 \%$ and $0.55 \%$ gains in PSNR and PSNR-HVS, but a loss of $0.92 \%$ and $0.02 \%$ in SSIM and MS-SSIM, respectively ${ }^{\ddagger}$. While extremely preliminary, these results show that even with minimal tuning, and without optimizing for the perceptual metrics for which it was designed, PVQ is competitive with scalar quantization. One potential drawback is that PVQ requires an extra forward transform to transform its predictor to the frequency domain. This was a cost we had already paid in Daala when using lapped transforms with frequency domain prediction, but is one we will have to justify in AV1.

\subsection{Chroma from Luma}

Chroma from Luma (CfL) has proved to be an effective tool to exploit local correlation between the color planes by building a linear model between the luma plane and each of the chroma planes in a given block. ${ }^{8}$ Frequency-domain CfL works particularly well in conjunction with PVQ: it allows separating off the DC coefficient (equivalent to the offset of the linear model) and the scale (equivalent to PVQ's gain parameter). Instead of trying to build a linear model implicitly without signaling, we find that simply using the luma coefficients directly as a shape predictor and signaling DC and gain parameters via normal means works even better. This reduced bitrate of the overall file by $1.4 \%$ for equal PSNR in the $\mathrm{Cb}$ plane and $0.4 \%$ for $\mathrm{Cr}^{\S}$. This is an example of how the flexibility of PVQ simplifies other coding tools.

The other advantage of operating in the frequency domain is that we can compute the predictor before performing final reconstruction of the pixels in the spatial domain. That reduces pipeline dependencies in a hardware implementation. For these reasons, we plan to investigate the integration of frequency-domain CfL into AV1 as soon as PVQ is working well.

\subsection{Directional Deringing}

Daala has a directional deringing filter that operates as an in-loop filter on the final reconstructed output of the encoder. $^{36}$ That makes it very straightforward to adapt to any other hybrid video codec, including AV1. We have already integrated it into AV1 as an experiment (--enable-dering) alongside the Constrained Low-Pass Filter (CLPF) from Thor (--enable-clpf). We tested both Daala's deringing filter and the CLPF, as well as applying both filters. For the latter, one filter runs and feeds its output into the other, in pipelined fashion. The results are summarized in Table 2, using both the low-latency and high-latency configurations of NETVC's proposed testing procedures. ${ }^{17}$

The larger gains for the low-latency configuration are expected, as the use of alt-refs in the high-latency configuration has a noise-removal and low-passing effect similar to that of the filters. We expect these gains will improve further, as the side information encoded for each superblock (to signal application of the filter and filter strength) is not currently entropy coded. When running both filters in sequence, each one currently makes decisions about whether or not to apply the filter and at what strength independently, while a joint decision might be better. Overall, these gains are smaller than when we tested the same filters in Daala, most likely because AV1 has less ringing to remove. Our general impression, however, is that the subjective improvements are still larger than the metrics would suggest.

\footnotetext{
${ }^{\dagger}$ On subset1 using commit 1ca568b15e4f for PVQ and commit 636b94545d51 for AV1, which enables trellis quantiza-

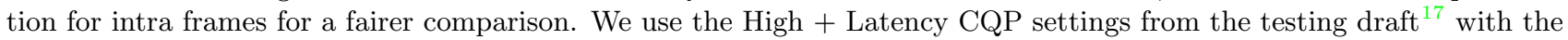
extra options --cpu-used $=1$ in both cases

${ }^{\ddagger}$ On objective-1-smc using commit 1ca568b15e4f for PVQ and commit 636b94545d51 for AV1 again, with the High Latency CQP settings from the testing draft ${ }^{17}$ with the extra options --cpu-used=1 --passes=1 --frame-parallel=1 in both cases.

${ }^{\S}$ See commit $0857 \mathrm{e} 3029854$ in the Daala git repository. Our other metrics are only designed to work on luma, which was only marginally affected by the change.
} 


\begin{tabular}{lrrrrrrrrr}
\hline & \multicolumn{2}{l}{ Low-latency } & & & \multicolumn{2}{l}{ High-latency } \\
\cline { 2 - 3 } Method & PSNR & PSR-HVS & SSIM & MS-SSIM & & PSNR & PSR-HVS & SSIM & MS-SSIM \\
\hline CLPF & $-2.80 \%$ & $-1.85 \%$ & $-2.77 \%$ & $-2.11 \%$ & & $-0.97 \%$ & $-0.33 \%$ & $-0.83 \%$ & $-0.48 \%$ \\
DD & $-3.54 \%$ & $-2.95 \%$ & $-3.95 \%$ & $-3.15 \%$ & & $-1.59 \%$ & $-0.95 \%$ & $-1.74 \%$ & $-1.17 \%$ \\
CLPF, then DD & $-3.87 \%$ & $-2.75 \%$ & $-3.97 \%$ & $-3.03 \%$ & & $-1.53 \%$ & $-0.55 \%$ & $-1.53 \%$ & $-0.85 \%$ \\
DD, then CLPF & $-4.01 \%$ & $-2.96 \%$ & $-4.16 \%$ & $-3.26 \%$ & & $-1.67 \%$ & $-0.73 \%$ & $-1.70 \%$ & $-1.03 \%$ \\
\hline
\end{tabular}

Table 2: Bitrate reductions for the Daala directional deringing filter (DD) and Thor's CLPF in AV1, in various configurations. All numbers are percent reductions in bitrate at equivalent quality for the given metric (more negative is better).

\begin{tabular}{lrrr}
\hline Method & VP9 & Daala & rANS \\
\hline Encoding & $107.2 \mathrm{Mbps}$ & $192.2 \mathrm{Mbps}$ & $366.8 \mathrm{Mbps}$ \\
Decoding & $112.6 \mathrm{Mbps}$ & $144.9 \mathrm{Mbps}$ & $181.5 \mathrm{Mbps}$ \\
\hline
\end{tabular}

Table 3: Encoder and decoder throughput for several entropy coding methods. All numbers are in megabits per second (higher is better).

Combining Daala's deringing with Thor's CLPF demonstrates that some gains are possible by using both, mostly in the low-latency configuration, but the current approach was done for simplicity, and will likely change before AV1 is done. Switching between the filters on a superblock basis instead of pipelining them would reduce the amount of line buffers required in hardware, but since they do not always operate on the same superblock size, this is not something we have attempted.

\subsection{Non-Binary Adaptive Entropy Coding}

Daala uses an adaptive entropy coder that, unlike most video codecs, is not restricted to coding symbols from binary alphabets. ${ }^{21}$ Entropy coding is an inherently serial process, and can be the limiting factor in lowering the clockrate required by a hardware implementation (and thus the power required). Every symbol clocked through the entropy coder incurs per-symbol overheads. The primary motivation of coding symbols from alphabets with more than two possible values is to reduce the number of symbols coded to improve hardware efficiency. By reducing the number of symbols we code, we can reduce these overheads as well as the number of serial dependencies.

\subsubsection{Non-Binary Coding}

We support alphabet sizes up to 16 using 15-bit probabilities. Larger alphabets are easy to support in software, but imposing a limit makes hardware simpler, and also allows SIMD implementations of probability updates. Thus each symbol coded with our non-binary coder can take the place of up to four binary symbols. This effectively gives us a (small) degree of parallelism.

Although we do not yet have a hardware implementation to use for comparisons, we created a small benchmark for testing software entropy coder implementations in isolation". Like VP9, AV1 codes symbols using binary trees, with a binary probability at each node of the tree. This benchmark randomly generates 100, 000, 000 symbols according to the default probability distribution of the tree used for $16 \times 16$ intra modes. It then converts the tree into a flat 15-bit cumulative distribution for use with our entropy coder and a flat 10-bit cumulative distribution for use with rANS, ${ }^{37}$ which is also under consideration for inclusion in AV1, and encodes and decodes each symbol with all three methods. The alphabet size is 10 and the average number of binary symbols required to encode a value from this distribution is 2.71 , with $51.5 \%$ of symbols requiring only a single binary symbol to code. The resulting throughput of the encoder and decoder in each method is given in Table 3.

\footnotetext{
ISee the ectest branch of Nathan E. Egge's personal AOM git repository: https://github.com/negge/aom/tree/ ectest
} 
This shows a significant speed-up for Daala over VP9, and rANS shows an even larger speed-up, particularly for encoding. However, rANS also brings several practical complications, such as the requirement of a $33 \mathrm{kbit}$ lookup table in its current implementation and the requirement that symbols be decoded in the opposite order from which they are encoded. The latter requires buffering all of the symbols for a tile in the encoder, which is expensive for hardware. We do not claim that these software implementations of each method are fully optimized, nor that this single distribution is representative of the entire codec, however we expect this general trend to hold. The exact speed-ups depend a good deal on the average number of binary symbols represented by a single non-binary symbol, where we feel there is significant room for improvement in the design of AV1.

\subsubsection{Adaptive Coding}

Currently, like VP9, AV1 uses static probabilities for an entire frame. These probabilities are initialized to default values, then automatically updated using the statistics of the previous frame, and optionally overridden by values explicitly coded in the frame header. In its current implementation, the AV1 encoder performs R-D optimization of the entire frame, recording all of its decisions. Then it uses the statistics of these decisions to compute optimal probabilities for that frame, and makes a R-D optimal decision about whether or not to encode an explicit override for each one.

This has several drawbacks for interactive applications. Because such applications have to be robust in the face of packet loss, they cannot use the feed-forward updates of the probabilities using the statistics from prior frames. That means all probability updates must be explicitly coded from the defaults for every frame. This adds bitrate overhead on the order of $5.5 \%$. It also means that the encoder must buffer the symbols to code for an entire frame, and cannot send out a single packet until the whole frame has finished encoding. As with rANS, this buffering is expensive. However, unlike rANS, it is possible to modify the implementation to encode with estimated probabilities rather than optimal probabilities. We have not modified the encoder to do so, but expect it would incur a similarly large overhead.

Instead, we are investigating using adaptive probabilities. Currently, Daala adapts most probabilities using simple frequency counts, periodically rescaling them as the total exceeds 15 bits. Since the total frequency count is generally not a power of two, we adapt the method of Stuiver and Moffat ${ }^{4}$ to coding these values without any multiplications or divisions (except that we over-estimate probabilities for symbols at the beginning of the alphabet, instead of the end). This method is only approximate, but has an overhead of around $1 \%$ to $2 \%$ compared to the theoretical entropy, which is similar to that of CABAC.

When the total frequency count is a power of two, we use $15 \times 16 \rightarrow 31$ multiplies to code symbols with negligible $(<0.01 \%)$ overhead. If we can adapt the probability distributions while leaving their total constant, we can avoid the overhead of the non-power-of-two approach. Let $f_{i}$ be a cumulative distribution of an alphabet of size $M$ with total $T$, such that $0<f_{0}<f_{1}<\ldots<f_{i}<\ldots<f_{M-1}=T$. Then, if we encode or decode symbol $j$, we propose updating the distribution $f_{i}$ with an update rate of $2^{r}$ using the following rule:

$$
f_{i}= \begin{cases}f_{i}-\left\lfloor\frac{f_{i}-i+2^{r}-2}{2^{r}}\right\rfloor, & i<j \\ f_{i}-\left\lfloor\frac{f_{i}-T+M-1-i}{2^{r}}\right\rfloor, & j \leq i\end{cases}
$$

This rule adjusts values of $f_{i}$ downward when $i<j$ and upward when $j \leq i$, but ensures that the difference between $f_{i}$ and $f_{i+1}$ is always at least 1 , and maintains the total, $T$. So long as the difference is larger than 1 , it can continue to adjust the corresponding probability, regardless of the rate, $2^{r}$. While this update rule looks much more expensive than using simple frequency counts, with tables built around the constants $M, T, 2^{r}, i$, and $j$, it can be reduced to two vector additions and a vector shift, which is comparable to a frequency count update and a rescaling operation.

We implemented this adaptation scheme, with some additional rules to speed up the adaptation of the first few symbols coded in each context. Just converting some of the symbols coded in Daala to use this scheme reduced bitrates by $0.3 \%$ to $0.4 \% \|$. Whether or not the complexity of the more expensive update rule is worth the reduction in entropy coder overhead is still an open question.

\footnotetext{
"See the dyadic_adapt8 branch of Jean-Marc Valin's personal Daala git repository: https://github.com/jmvalin/ daala/tree/exp_dyadic_adapt8
} 
We think that some of these ideas merit inclusion in AV1, though which exact direction the Alliance ultimately goes depends on exact engineering trade-offs and implementation costs, especially in hardware. We will continue to experiment with these ideas and get more feedback from our partners in the Alliance.

\subsection{Rate Control}

Daala extends a bit-reservoir rate control system refined from the Vorbis audio codec and Theora video codec. The rate control is conceptually simple, flexible, stable, precise, and adapts quickly.

\subsubsection{Rate Control Model}

Daala controls rate by manipulating the quantization parameter, and thus quantizers, used in encoding. A simple model predicts bit usage for a given frame for any quantizer.

$$
R=\text { scale } \cdot Q^{-\alpha}
$$

$R$ represents the rate of a frame in bits, $Q$ is the actual linear quantizer (a function of the quantization parameter), and $\alpha$ is a fixed modeling exponent chosen for an entire sequence based on frame type and target rate (in bits-per-pixel). We measure the parameter scale for each frame type during encoding.

\subsubsection{Estimating scale}

After encoding a frame, we know $R$ and $Q$, and $\alpha$ is fixed. As a result we can solve the above relationship for scale.

We smooth this measured scale value using a digital approximation of a second order Bessel filter. The filter damps noise and oscillations while preserving reaction speed (see Figure 6), using a time constant that allows full-scale reaction in half the buffer interval. We adjust the reaction time to allow faster adaptation at the beginning of a sequence.

Daala models a separate scale parameter for each frame type and so maintains a Bessel filter for each of keyframes, long-term reference (golden) frames, regular P frames, and B frames.

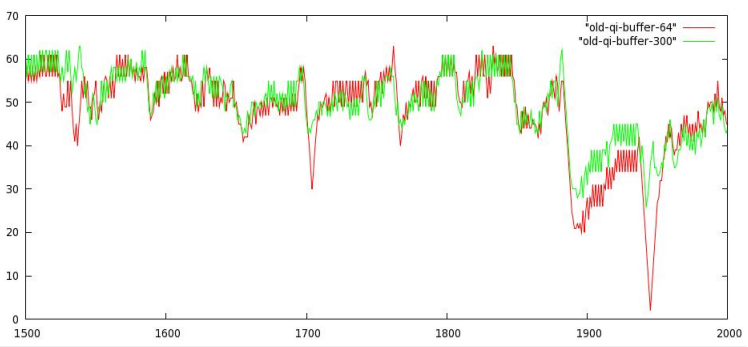

(a) Exponential Moving Average

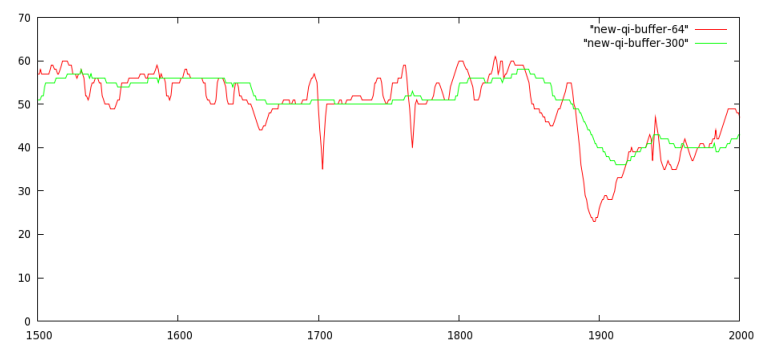

(b) $2^{\text {nd }}$ Order Bessel Filter

Figure 6: (a) Smoothing scale modeling and quantizer choice using an exponential moving average produces unstable/oscillatory behavior detrimental to objective and visual quality. (b) A $2^{\text {nd }}$ order Bessel filter damps the oscillations while preserving reaction speed. In addition, the Bessel filter's time constant may be adjusted independent of buffer size to produce any desired reaction speed.

\subsubsection{Choosing $Q$}

For every frame, Daala constructs a bit usage plan for the entire remaining buffer interval by predicting the frame type of each subsequent frame and summing the bits used for each of these frames according to our prediction model. The goal of the bit usage plan is to hit a fixed buffer fullness level at the last keyframe in the buffer interval. 


$$
R_{\text {total }}=\sum_{i} N_{i} \cdot \text { scale }_{i} \cdot Q_{i}^{-\alpha}
$$

Here, $N_{i}$ is the number of frames of each type, scale ${ }_{i}$ are the Bessel-smoothed scales corresponding to each frame type, and quantizers $Q_{i}$ are functions of the quantization parameter. The encoder simply searches quantization parameters until $R_{\text {total }}$ hits the desired bit usage target.

After encoding each frame, we discard the plan and construct a new plan starting from the next frame.

\subsubsection{Two-Pass Rate Control}

Most modern video encoders offer two-pass rate control which makes a first pass over a video file to collect metrics, and performs encoding in a second pass using knowledge of the complete video content. Daala's two-pass rate control is a simple modification of the (one-pass, causal) rate control described thus far.

Rather than smoothing scale values for each frame in a Bessel filter, we simply measure and save the scale values during the first pass, and sum these empirical measured values during second pass encoding to use in place of the product $N_{i} \cdot$ scale $_{i}$ in equation 9 . We also add a fixed offset to correct for consistent over/under estimates. Everything else is just as in one-pass.

\subsubsection{Chunked Two-Pass}

Video hosting services and sites such as YouTube typically encode files by splitting them into many small chunks of one to five seconds each, and then encoding each chunk independently in parallel to reduce encoding time.

Daala's rate control model allows the collection of complete file metrics from each chunk in a first pass, allowing the second pass encoding of each chunk to take into account the entire sequence. This produces a chunked two-pass rate control with quality performance similar or identical to a monolithic two-pass encoding.

We plan to investigate integration of this rate control method as an alternative to AV1's existing rate control. We do not yet know which approach will prove more effective, but conceptually Daala's is simple and easily adaptable to a broad array of use cases.

\section{CONCLUSION}

Overall, the Daala project has produced many important lessons and many promising technologies. Given that most of these are new or relatively unproven, we consider the results we were able to obtain with them encouraging. These results have steadily improved for the past several years (see Figure 7), and the overall Daala design may ultimately prove viable in the longer term. Several of the individual tools are also under active consideration for inclusion in AV1, the Alliance for Open Media's first video codec. We will continue to improve them as development of AV1 proceeds, with the hope of creating a truly state-of-the-art royalty-free codec.

\section{ACKNOWLEDGMENTS}

The authors would like to thank Steinar Midtskogen for his assistance in generating the deringing and CLPF results, as well as James Bankoski, Alex Converse, and Yaowu Xu for their assistance integrating code into AV1, in addition to all of the other volunteers that contributed to the open-source Daala project. 


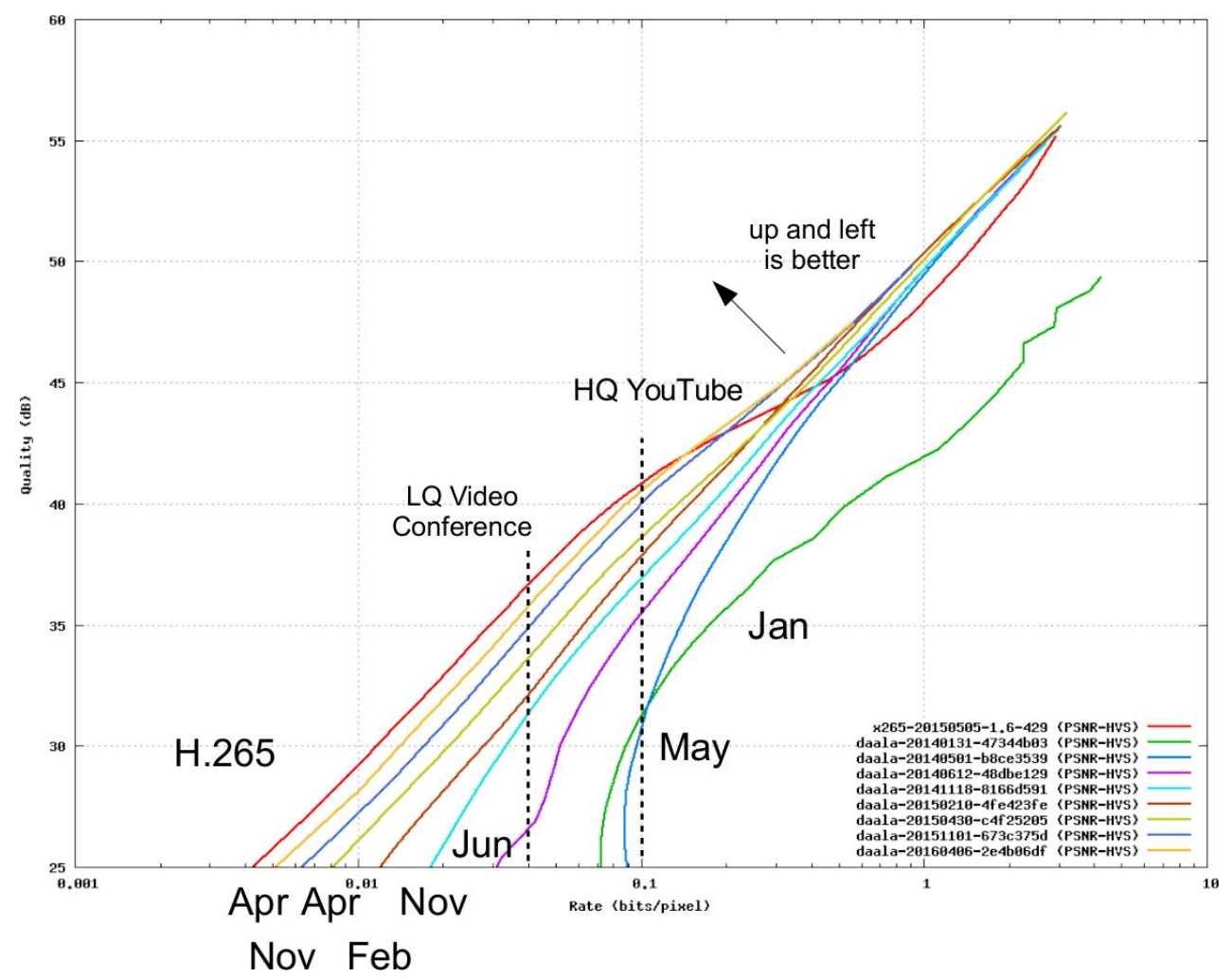

(a) PSNR-HVS-M

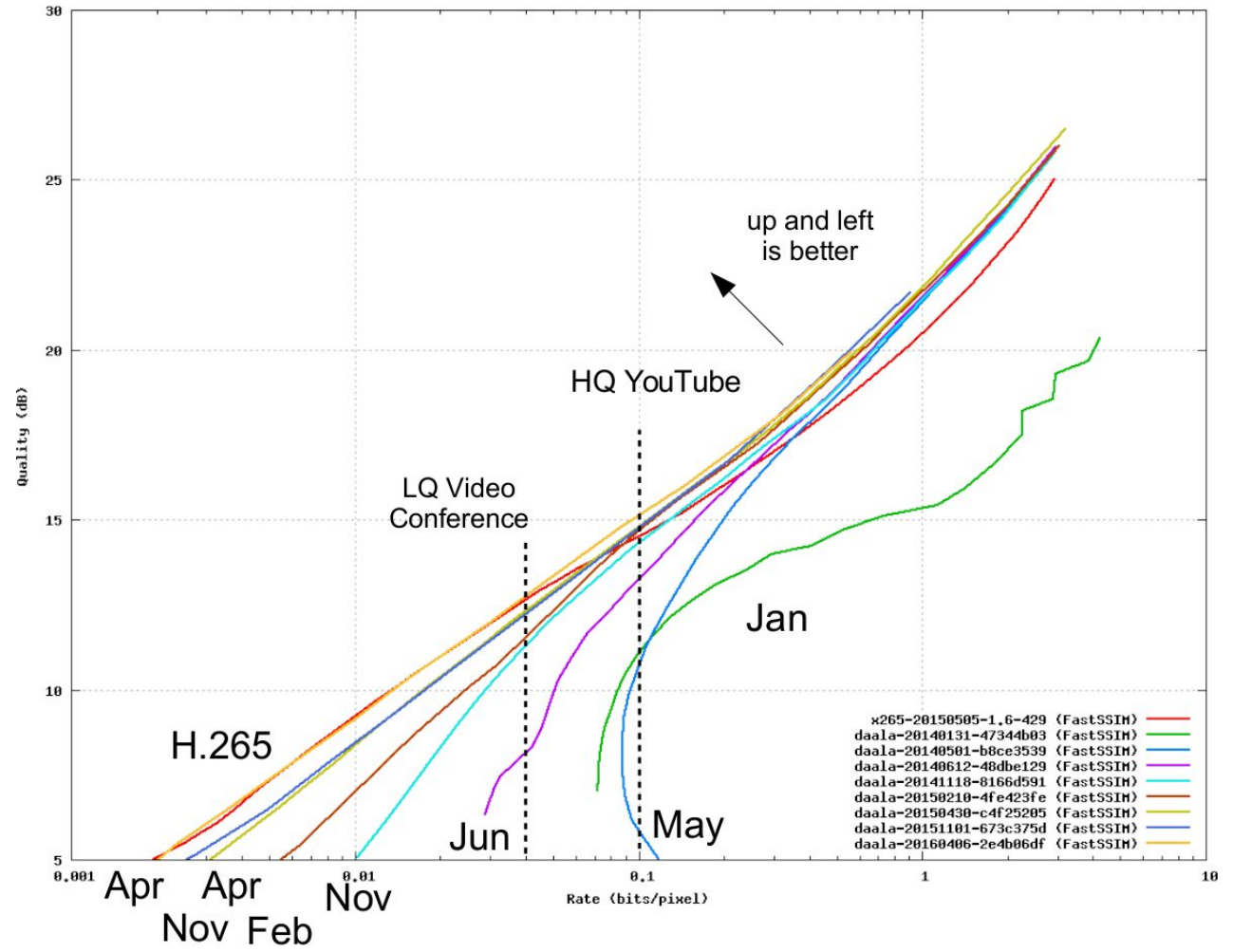

(b) Multiscale FastSSIM

Figure 7: Progress of the Daala project vs. x265 from Jaunary 2014 to April 2016. 


\section{REFERENCES}

[1] Reader, C., "Technical design \& IPR analysis for royalty-free video codecs," in [Proceedings of the $28^{\text {th }}$ Picture Coding Symposium (PCS'10)], 102-105 (Dec. 2010).

[2] "Daala website." https://xiph.org/daala/.

[3] Watanabe, H. and Singhal, S., "Windowed motion compensation," in [SPIE Visual Communications and Image Processing '91], 1605, 582-589 (Nov. 1991).

[4] Stuiver, L. and Moffat, A., "Piecewise integer mapping for arithmetic coding," in [Proceedings of the IEEE Data Compression Conference], 1-10 (Mar./Apr. 1998).

[5] Tran, T. D., "Lapped transform via time-domain pre- and post-filtering," IEEE Transactions on Signal Processing 51, 1556-1571 (May 2001).

[6] Terriberry, T. B., "Adaptive motion compensation without blocking artifacts," in [Proceedings of Visual Information Processing and Communication VI], Said, A., Guleryuz, O. G., and Stevenson, R. L., eds., 9410 (Mar. 2015).

[7] Valin, J.-M. and Terriberry, T. B., "Perceptual vector quantization for video coding," in [Proceedings of Visual Information Processing and Communication VI], Said, A., Guleryuz, O. G., and Stevenson, R. L., eds., 9410 (Mar. 2015).

[8] Egge, N. E. and Valin, J.-M., "Predicting chroma from luma with frequency domain intra prediction," in [Proceedings of Visual Information Processing and Communication VI], 9410 (Mar. 2015).

[9] Grange, A., de Rivaz, P., and Hunt, J., "VP9 bitstream \& decoding process specification version 0.6." https://storage.googleapis.com/downloads.webmproject.org/docs/vp9/ vp9-bitstream-specification-v0.6-20160331-draft.pdf (Mar. 2016).

[10] "Daala git repository." https://git.xiph.org/daala.git.

[11] "Alliance for open media git repository." https://aomedia.googlesource.com/aom.

[12] "Are we compressed yet? website." https://arewecompressedyet.com/.

[13] Wang, Z., Bovik, A. C., Sheikh, H. R., and Simoncelli, E. P., "Image quality assessment: From error visibility to structural similarity," IEEE Transactions on Image Processing 13, 600-612 (Apr. 2004).

[14] Ponomarenko, N., Silvestri, F., Egiazarian, K., Carli, M., Astola, J., and Lukin, V., "On between-coefficient contrast masking of DCT basis functions," in [CD-ROM Proceedings of the $3^{\text {rd }}$ International Workshop on Video Processing and Quality Metrics for Consumer Electronics (VPQM '07)], (Jan. 2007).

[15] Chen, M.-J. and Bovik, A. C., "Fast structural similarity index algorithm," Journal of Real-Time Image Processing 6, 281-287 (Dec. 2011).

[16] Wang, Z., Simoncelli, E. P., and Bovik, A. C., "Multi-scale structural similarity for image quality assessment," in [Proceedings of the $37^{\text {th }}$ IEEE Conference on Signals, Systems, and Computers], 2, 1398-1402 (Nov. 2003).

[17] Daede, T. J., Norkin, A., and Brailovskiy, I., "Video codec testing and quality measurement." https: //tools.ietf.org/html/draft-ietf-netvc-testing (July 2016).

[18] "Are we compressed yet? git repository." https://github.com/tdaede/awcy.

[19] "rd_tool git repository." https://github.com/tdaede/rd_tool.

[20] Egge, N. E., "Time domain lapped transforms for video coding." https://tools.ietf.org/html/ draft-egge-netvc-tdlt (2015).

[21] Terriberry, T. B., "Coding tools for a next generation video codec." https://tools.ietf.org/html/ draft-terriberry-netvc-coding-tools (2015).

[22] Dai, W., Liu, L., and Tran, T. D., "Adaptive block-based image coding with pre-/post-filtering," in [Proceedings of the $15^{\text {th }}$ Data Compression Conference (DCC'05)], 73-82 (Mar. 2005).

[23] Daede, T. J., Egge, N. E., Valin, J.-M., Martres, G., and Terriberry, T. B., "Daala: A perceptuallydriven next generation vidoe codec." Presented at the $26^{\text {th }}$ Data Compression Conference (DCC'16), https : //arxiv.org/abs/1603.03129 (Mar. 2016).

[24] Fuldseth, A., Bjontegaard, G., Midtskogen, S., Davies, T., and Zanaty, M., "Thor video codec." https: //tools.ietf.org/html/draft-fuldseth-netvc-thor (Mar. 2016). 
[25] de Oliveira, R. G. and Pesquet-Popescu, B., "Intra-frame prediction with lapped transforms for image coding," in [Prooceedings of the $36^{\text {th }}$ IEEE International Conference on Acoustics, Speech, and Signal Processing (ICASSP'11)], 805-808 (May 2011).

[26] Xu, J., Wu, F., and Zhang, W., "Intra-predictive transforms for block-based image coding," IEEE Transactions on Signal Processing 57, 3030-3040 (Aug. 2009).

[27] Sezer, O. G., Harmanci, O., and Guleryuz, O. G., "Sparse orthonormal transforms for image compression," in [Proceedings of the $15^{\text {th }}$ IEEE International Conference on Image Processing (ICIP'08)], 149-152 (Oct. 2008).

[28] Bankoski, J., Koleszar, J., Quillio, L., Salonen, J., Wilkins, P., and Xu, Y., "RFC 6386 - VP8 data format and decoding guide." https://tools.ietf.org/html/rfc6386 (Nov. 2011).

[29] Valin, J.-M., Maxwell, G., Terriberry, T. B., and Vos, K., "High-quality, low-delay music coding in the Opus codec," in [Proceedings of the $135^{\text {th }}$ Audio Engineering Society Convention], (Oct. 2013).

[30] Fukuma, S., Oyama, K., Iawahashi, M., and Kambayashi, N., "Lossless 8-point fast discrete cosin transform using lossless Hadamard transform," tech. rep., The Institute of Electronics, Information, and Communication Engineers of Japan (Oct. 1999).

[31] Tu, C., Srinivasan, S., Sullivan, G. J., Regunathan, S., and Malvar, H. S., [Low-Complexity Hierarchical Lapped Transform for Lossy-to-Lossless Image Coding in JPEG XR/HD Photo], vol. 7073 of Proceedings of the SPIE (Aug. 2008).

[32] Valin, J.-M., Terriberry, T. B., Egge, N. E., Daede, T. J., Cho, Y., Montgomery, C., and Bebenit, M., "Daala: Building a next-generation video codec from unconventional technology," in [Proceedings of the $18^{\text {th }}$ IEEE Multimedia Signal Processing Workshop (MMSP'16) (to appear)], (Sept. 2016).

[33] Tran, T. D. and Tu, C., [Lapped Transform Based Video Coding], vol. 4472 of Proceedings of the SPIE (July 2001).

[34] Mukherjee, D., Su, H., Bankoski, J., Converse, A., Han, J., Liu, Z., and Xu, Y., [An Overview of New Video Coding Tools Under Consideration for VP10: the Successor to VP9], vol. 9599 of Proceedings of the SPIE (Aug. 2015).

[35] Han, J., Xu, Y., and Mukherjee, D., "A butterfly structured design of the hybrid transform coding scheme," in [Proceedings of the $30^{\text {th }}$ Picture Coding Symposium (PCS'13)], 17-20 (Dec. 2013).

[36] Valin, J.-M., "The Daala directional deringing filter." https://arxiv.org/pdf/1602.05975.pdf (Apr. 2016).

[37] Duda, J., Tahboub, K., Gadgil, N. J., and Delp, E. J., "The use of asymmetric numeral systems as an accurate replacement for Huffman coding," in [Proceedings of the $31^{\text {st }}$ Picture Coding Symposium (PCS'15)], 65-69 (May 2015). 